\title{
SCATTERED RADIATION IN THE OZONE ABSORPTION BANDS AT SELECTED LEVELS OF A TERRESTRIAL, RAYLEIGH ATMOSPHERE
}

\author{
J. V. Dave and P. M. Furukawa \\ (Meteor. Monogr., 7, No. 29, Amer. Met. Soc., Boston, 1966)
}

A computer programming error, discovered after the publication of the monograph, has resulted in incorrect values of $I_{c}^{*} / Q$ and $I_{r}^{*} / Q$ of the Upward Radiation being listed in Tables 1 through 16. The error arose because the factor $3 / 16 \mu$ in Eq. (26) (see text of monograph) was programmed as $3 \mu / 16$. Since the factor is associated with only one of the terms on the right-hand side of the equation, the values of $I_{e}^{*} / Q$ and $I_{r}^{*} / Q$ of the Upward Radiation listed in the tables need to be multiplied by a factor somewhat less than $1 / \mu^{2}$. The values $I_{e}^{*} / Q$ and $I_{r}^{*} / Q$ of the Upward Radiation are correct at all pressure levels for the nadir direction $\left(\theta=0^{\circ}\right)$ since $\mu=1$ for this direction of observation.

The appended tables were computed to provide a method for determining the correct values of the reflected component of the upward radiation. In order to keep the size of the errata tables to a minimum without requiring the user to do any more computation than before, values of $I_{e}^{*} / I_{g}$ and $I_{r}^{*} / I_{y}$ are tabulated instead of $I_{e}^{*} / Q$ and $I_{r}^{*} / Q$ as was done originally. It will be seen from Eqs. (26) and (30) that the ratios now tabulated are independent of $\theta_{0}$. The procedure for using the appended tables is as follows:

1. The value of $Q$ for a given $\lambda$ and a desired $R$ must first be determined from Eq. (31), using the values of $S^{\mathbf{b}}\left(\tau_{1}\right)$ given in Table B of the monograph.

2. The $Q$ thus obtained, when multiplied by the appropriate value of $I_{g} / Q$ (also obtained from Table B), then gives $I_{l}$ for a given $\theta_{0}$.

3. Next, the value of $I_{y}$ from the step above should be multiplied by the values of $I_{c}^{*} / I_{y}$ and $I_{r}^{*} / I_{y}$ given in the appended tables to get $I_{e}^{*}$ and $I_{r}^{*}$.

4. The values of $I_{r}^{*}$ and $I_{r}^{*}$ can then be applied in the manner described in Sec.C.1. (p. 9) in the monograph.

Acknowledgement. We are indebted to our colleague, Dr. Carl L. Mateer, for bringing the discrepancy in the tables to our attention and for his assistance in resolving the problem. 
Parameters for computing the reflected component of the Upward Radiation

\begin{tabular}{|c|c|c|c|c|c|c|c|c|c|}
\hline \multirow{2}{*}{$\begin{array}{l}\text { press. } \\
\text { level } \\
(\mathrm{mb})\end{array}$} & \multirow{2}{*}{$\theta$} & \multicolumn{2}{|c|}{$\lambda=2875.0 \AA$} & \multicolumn{2}{|c|}{$\lambda=2925.0 \AA$} & \multicolumn{2}{|c|}{$\lambda=2975.0 \AA$} & \multicolumn{2}{|c|}{$\lambda=3025.0 \AA$} \\
\hline & & & & & & & & & \\
\hline \multirow[t]{3}{*}{0} & $\begin{array}{l}0 \\
15 \\
30 \\
40 \\
50\end{array}$ & $\begin{array}{l}8.590-09 \\
4.605 \\
5.692-10 \\
4.570-11 \\
3.091-12\end{array}$ & $\begin{array}{l}8.590-09 \\
4.648 \\
5.970-10 \\
5.294-11 \\
6.027-12\end{array}$ & $\begin{array}{l}4.747-05 \\
3.438 \\
1.163 \\
3.040-06 \\
3.721-07\end{array}$ & $\begin{array}{l}4.747-05 \\
3.465 \\
1.206 \\
3.301-06 \\
4.670-07\end{array}$ & $\begin{array}{l}2.752-03 \\
2.296 \\
1.251 \\
5.887-04 \\
1.751\end{array}$ & $\begin{array}{l}2.752-03 \\
2.310 \\
1.286 \\
6.235-04 \\
1.976\end{array}$ & $\begin{array}{l}2.892-02 \\
2.621 \\
1.883 \\
1.251 \\
6.473-03\end{array}$ & $\begin{array}{l}2.892-02 \\
2.632 \\
1.920 \\
1.301 \\
6.971-03\end{array}$ \\
\hline & 60 & 1.309 & 4.038 & $3.300-08$ & $7.701-08$ & $2.494-05$ & $3.469-05$ & 2.155 & 2.511 \\
\hline & $\begin{array}{l}70 \\
75 \\
80 \\
85\end{array}$ & $\begin{array}{l}7.365-13 \\
5.705 \\
4.699 \\
4.547\end{array}$ & $\begin{array}{l}3.642 \\
3.645 \\
3.827 \\
4.457\end{array}$ & $\begin{array}{l}1.423 \\
1.159 \\
1.028 \\
1.060\end{array}$ & $\begin{array}{l}5.409 \\
5.290 \\
5.503 \\
6.356\end{array}$ & $\begin{array}{l}3.050-06 \\
2.152 \\
1.907 \\
2.009\end{array}$ & $\begin{array}{l}8.337-06 \\
7 \cdot 166 \\
7 \cdot 143 \\
8 \cdot 135\end{array}$ & $\begin{array}{l}3.278-04 \\
1.123 \\
6.888-05 \\
6.782\end{array}$ & $\begin{array}{l}5.024-04 \\
2.383 \\
1.807 \\
1.909\end{array}$ \\
\hline \multirow[t]{2}{*}{0.18} & $\begin{array}{r}0 \\
15 \\
30 \\
40 \\
50\end{array}$ & $\begin{array}{l}8.672-09 \\
4 \cdot 651 \\
5 \cdot 754-10 \\
4 \cdot 620-11 \\
3.075-12\end{array}$ & $\begin{array}{l}8.672-09 \\
4.694 \\
6.035-10 \\
5.349-11 \\
5.984-12\end{array}$ & $\begin{array}{l}4.770-05 \\
3.455 \\
1 \cdot 170 \\
3.059-06 \\
3.744-07\end{array}$ & $\begin{array}{l}4 \cdot 770-05 \\
3 \cdot 482 \\
1 \cdot 213 \\
3 \cdot 321-06 \\
4 \cdot 693-07\end{array}$ & $\begin{array}{l}2.759-03 \\
2 \cdot 302 \\
1 \cdot 255 \\
5.907-04 \\
1.758\end{array}$ & $\begin{array}{l}2.759-03 \\
2 \cdot 316 \\
1 \cdot 290 \\
6.256-04 \\
1.983\end{array}$ & $\begin{array}{l}2.896-02 \\
2.624 \\
1.886 \\
1.253 \\
6.487-03\end{array}$ & $\begin{array}{l}2.896-02 \\
2.636 \\
1.923 \\
1.303 \\
6.985-03\end{array}$ \\
\hline & $\begin{array}{l}60 \\
70 \\
75 \\
80 \\
85\end{array}$ & $\begin{array}{l}1.279 \\
7.068-13 \\
5.399 \\
4.350 \\
4.019\end{array}$ & $\begin{array}{l}3.944 \\
3.493 \\
3.447 \\
3.540 \\
3.936\end{array}$ & $\begin{array}{l}3.276-08 \\
1.386 \\
1.117 \\
9.751-09 \\
9.720\end{array}$ & $\begin{array}{l}7.624-08 \\
5.264 \\
5.095 \\
5.217 \\
5.825\end{array}$ & $\begin{array}{l}2.502-05 \\
3.014-06 \\
2.103 \\
1.840 \\
1.891\end{array}$ & $\begin{array}{l}3.475-05 \\
8.218-06 \\
6.995 \\
6.887 \\
7.652\end{array}$ & $\begin{array}{l}2.160 \\
3.280-04 \\
1.115 \\
6.750-05 \\
6.520\end{array}$ & $\begin{array}{l}2.516 \\
5.018-04 \\
2.360 \\
1.768 \\
1.834\end{array}$ \\
\hline \multirow[t]{3}{*}{1.40} & $\begin{array}{r}0 \\
15 \\
30 \\
40 \\
50\end{array}$ & $\begin{array}{l}1.026-08 \\
5.532-09 \\
6.978-10 \\
5.694-11 \\
3.464-12\end{array}$ & $\begin{array}{l}1.026-08 \\
5.584-09 \\
7.317-10 \\
6.567-11 \\
6.644-12\end{array}$ & $\begin{array}{l}5.190-05 \\
3.770 \\
1.289 \\
3.411-06 \\
4.224-07\end{array}$ & $\begin{array}{l}5.190-05 \\
3.799 \\
1.337 \\
3.701-06 \\
5.262-07\end{array}$ & $\begin{array}{l}2.887-03 \\
2.413 \\
1.322 \\
6.265-04 \\
1.883\end{array}$ & $\begin{array}{l}2.887-03 \\
2.428 \\
1.359 \\
6.634-04 \\
2.122\end{array}$ & $\begin{array}{l}2.965-02 \\
2.688 \\
1.937 \\
1.292 \\
6.722-03\end{array}$ & $\begin{array}{l}2.965-02 \\
2.700 \\
1.975 \\
1.343 \\
7.235-03\end{array}$ \\
\hline & 60 & 1.304 & 4.008 & $3.443-08$ & $7.849-08$ & $2.702-05$ & $3.714-05$ & $2 \cdot 258$ & 2.624 \\
\hline & $\begin{array}{l}70 \\
75 \\
80 \\
85\end{array}$ & $\begin{array}{l}6.682-13 \\
4.848 \\
3.612 \\
2.904\end{array}$ & $\begin{array}{l}3.287 \\
3.078 \\
2.919 \\
2.822\end{array}$ & $\begin{array}{l}1.309 \\
1.008 \\
8.278-09 \\
7.318\end{array}$ & $\begin{array}{l}4 \cdot 936 \\
4 \cdot 567 \\
4 \cdot 395 \\
4 \cdot 350\end{array}$ & $\begin{array}{l}2.988-06 \\
1.963 \\
1.620 \\
1.511\end{array}$ & $\begin{array}{l}8.005-06 \\
6.467 \\
6.015 \\
6.068\end{array}$ & $\begin{array}{l}3 \cdot 432-04 \\
1 \cdot 120 \\
6 \cdot 281-05 \\
5 \cdot 547\end{array}$ & $\begin{array}{l}5.181-04 \\
2.325 \\
1.629 \\
1.549\end{array}$ \\
\hline \multirow[t]{2}{*}{6.40} & $\begin{array}{l}0 \\
15 \\
30 \\
40 \\
50\end{array}$ & $\begin{array}{l}4.651-08 \\
2.646 \\
3.987-09 \\
3.958-10 \\
2.055-11\end{array}$ & $\begin{array}{l}4.651-08 \\
2.670 \\
4.177-09 \\
4.503-10 \\
3.615-11\end{array}$ & $\begin{array}{l}1.106-04 \\
8.252-05 \\
3.085 \\
9 \cdot 116-06 \\
1.321\end{array}$ & $\begin{array}{l}1.106-04 \\
8.316-05 \\
3.198 \\
9.869-06 \\
1.609\end{array}$ & $\begin{array}{l}4.330-03 \\
3.671 \\
2.110 \\
1.062 \\
3.514-04\end{array}$ & $\begin{array}{l}4.330-03 \\
3.694 \\
2.168 \\
1.123 \\
3.940-04\end{array}$ & $\begin{array}{l}3.637-02 \\
3.322 \\
2.453 \\
1.686 \\
9.219-03\end{array}$ & $\begin{array}{l}3.637-02 \\
3.336 \\
2.500 \\
1.751 \\
9.904-03\end{array}$ \\
\hline & $\begin{array}{l}60 \\
70 \\
75 \\
80 \\
85\end{array}$ & $\begin{array}{l}5.246-12 \\
2.242 \\
1.491 \\
1.034 \\
8.803-13\end{array}$ & $\begin{array}{l}1.575 \\
1.064 \\
9.049-12 \\
7.895 \\
8.012\end{array}$ & $\begin{array}{l}9.760-08 \\
2.535 \\
1.741 \\
1.253 \\
9.728-09\end{array}$ & $\begin{array}{l}1.991-07 \\
9.113-08 \\
7.479 \\
6.279 \\
5.441\end{array}$ & $\begin{array}{l}5.773-0.5 \\
5.395-06 \\
2.876 \\
2.025 \\
1.535\end{array}$ & $\begin{array}{l}7.647-05 \\
1.306 \\
8.907-06 \\
7.100 \\
5.835\end{array}$ & $\begin{array}{l}3.366 \\
5.736-04 \\
1.764 \\
7.816-05 \\
5.398\end{array}$ & $\begin{array}{l}3.882 \\
8.231-04 \\
3.342 \\
1.907 \\
1.437\end{array}$ \\
\hline
\end{tabular}




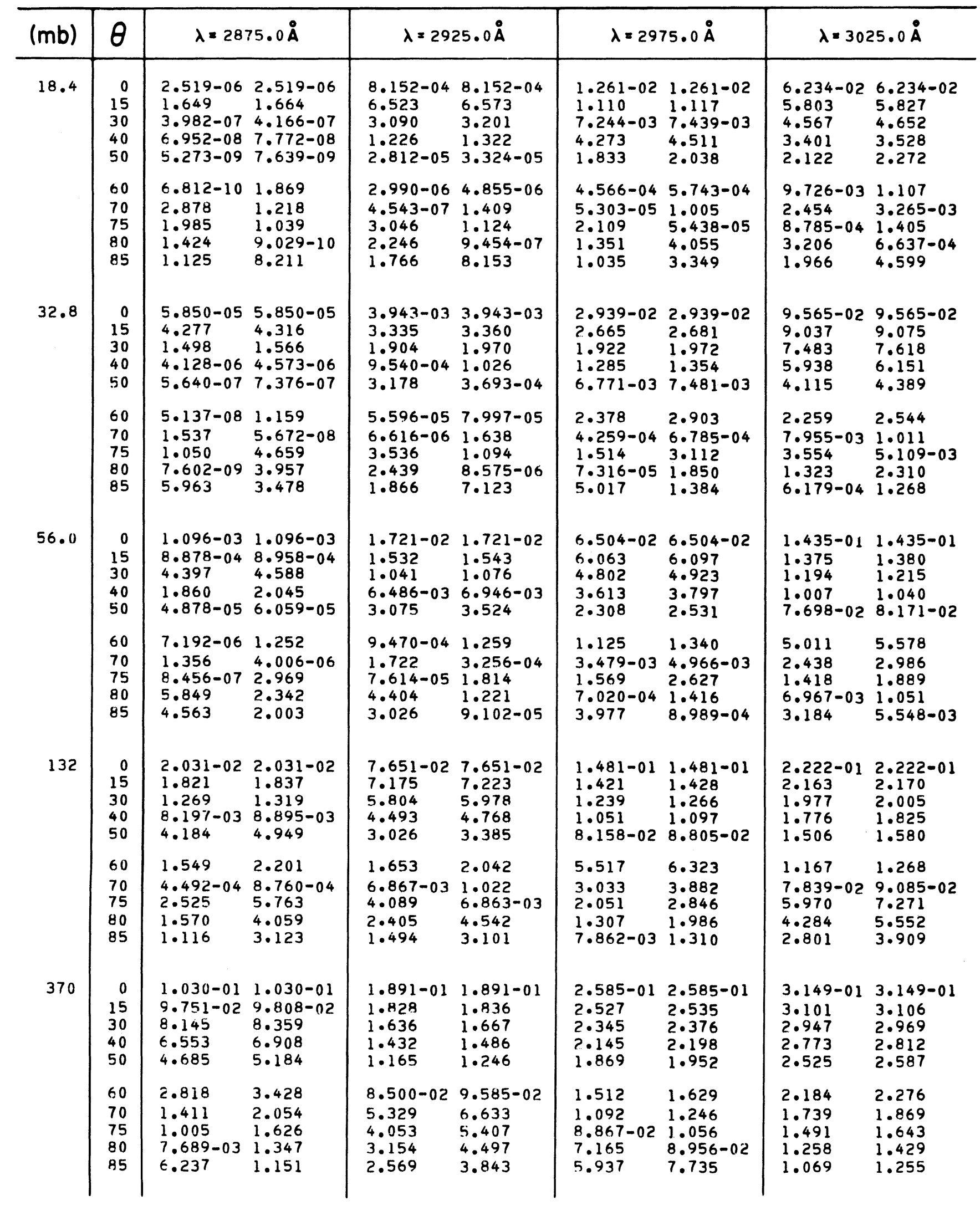


Parameters for computing the reflected component of the Upward Radiation

\begin{tabular}{|c|c|c|c|c|c|c|c|c|c|}
\hline \multirow{2}{*}{$\begin{array}{l}\text { press. } \\
\text { level } \\
\text { (mb) }\end{array}$} & \multirow{2}{*}{$\theta$} & \multicolumn{2}{|c|}{$\lambda=3075.0 \AA$} & \multicolumn{2}{|c|}{$\lambda=3125.0 \AA$} & \multicolumn{2}{|c|}{$\lambda=3175.0 \AA$} & \multicolumn{2}{|c|}{$\lambda=3225.0 \AA$} \\
\hline & & $I_{*}^{*} / I_{0}$ & $I_{r}^{*} / I_{g}$ & $I_{0}^{*} / I_{0}$ & $I_{r}^{*} / I_{g}$ & $I_{0}^{*} / I_{g}$ & & $I_{*}^{*} / I_{g}$ & \\
\hline \multirow[t]{4}{*}{0} & $\begin{array}{r}0 \\
15 \\
30 \\
40 \\
50\end{array}$ & $\begin{array}{l}9.257-02 \\
8.738 \\
7.204 \\
5.679 \\
3.877\end{array}$ & $\begin{array}{l}9.257-02 \\
8.764 \\
7.300 \\
5.828 \\
4.068\end{array}$ & $\begin{array}{l}1.779-01 \\
1.719 \\
1.531 \\
1.329 \\
1.061\end{array}$ & $\begin{array}{l}1.779-01 \\
1.722 \\
1.544 \\
1.351 \\
1.093\end{array}$ & $\begin{array}{l}2.351-01 \\
2.293 \\
2.111 \\
1.908 \\
1.626\end{array}$ & $\begin{array}{l}2.351-01 \\
2.296 \\
2.124 \\
1.930 \\
1.661\end{array}$ & $\begin{array}{l}2.779-01 \\
2.727 \\
2.560 \\
2.370 \\
2.100\end{array}$ & $\begin{array}{l}2.779-01 \\
2.729 \\
2.571 \\
2.390 \\
2.132\end{array}$ \\
\hline & 60 & 2.051 & 2.246 & $7.310-02$ & $7.728-02$ & $1 \cdot 251$ & $1 \cdot 301$ & 1.724 & 1.773 \\
\hline & $\begin{array}{l}70 \\
75\end{array}$ & $\begin{array}{l}6.458-03 \\
2.557\end{array}$ & $\begin{array}{l}7.886-03 \\
3.589\end{array}$ & $\begin{array}{l}3.732 \\
2.122\end{array}$ & $\begin{array}{l}4.175 \\
2.520\end{array}$ & $\begin{array}{l}7.852-02 \\
5.326\end{array}$ & $\begin{array}{l}8.482-02 \\
5.980\end{array}$ & $\begin{array}{l}1.221 \\
9.217-02\end{array}$ & $\begin{array}{l}1.291 \\
1.002\end{array}$ \\
\hline & $\begin{array}{l}80 \\
85\end{array}$ & $\begin{array}{l}8.916-04 \\
5.879\end{array}$ & $\begin{array}{l}1.620 \\
1.251\end{array}$ & $\begin{array}{l}9.087-03 \\
3.427\end{array}$ & $\begin{array}{l}1.217 \\
5.641-03\end{array}$ & $\begin{array}{l}2.920 \\
1.118\end{array}$ & $\begin{array}{l}3.520 \\
1.563\end{array}$ & $\begin{array}{l}5.997 \\
2.814\end{array}$ & $\begin{array}{l}6.851-02 \\
3.550\end{array}$ \\
\hline \multirow[t]{3}{*}{0.18} & $\begin{array}{r}0 \\
15 \\
30 \\
40 \\
50\end{array}$ & $\begin{array}{l}9.265-02 \\
8.745 \\
7.210 \\
5.684 \\
3.881\end{array}$ & $\begin{array}{l}9.265-02 \\
8.771 \\
7.306 \\
5.834 \\
4.072\end{array}$ & $\begin{array}{l}1.780-01 \\
1.719 \\
1.532 \\
1.330 \\
1.061\end{array}$ & $\begin{array}{l}1.780-01 \\
1.723 \\
1.545 \\
1.352 \\
1.094\end{array}$ & $\begin{array}{l}2.351-01 \\
2.294 \\
2.112 \\
1.909 \\
1.627\end{array}$ & $\begin{array}{l}2.351-01 \\
2 \cdot 297 \\
2 \cdot 124 \\
1.931 \\
1.662\end{array}$ & $\begin{array}{l}2.780-01 \\
2.727 \\
2.561 \\
2.371 \\
2.100\end{array}$ & $\begin{array}{l}2.780-01 \\
2.730 \\
2.571 \\
2.391 \\
2.132\end{array}$ \\
\hline & 60 & 2.054 & 2.249 & $7.316-02$ & $7.734-02$ & $1 \cdot 252$ & 1.302 & 1.725 & 1.774 \\
\hline & $\begin{array}{l}70 \\
75 \\
80 \\
85\end{array}$ & $\begin{array}{l}6.468-03 \\
2.558 \\
8.858-04 \\
5.739\end{array}$ & $\begin{array}{l}7.894-03 \\
3.586 \\
1.606 \\
1.220\end{array}$ & $\begin{array}{l}3.736 \\
2.124 \\
9.085-03 \\
3.390\end{array}$ & $\begin{array}{l}4.178 \\
2.522 \\
1.215 \\
5.576-03\end{array}$ & $\begin{array}{l}7.858-02 \\
5.331 \\
2.921 \\
1.113\end{array}$ & $\begin{array}{l}8.487-02 \\
5.984 \\
3.520 \\
1.555\end{array}$ & $\begin{array}{l}1.222 \\
9.222-02 \\
6.000 \\
2.810\end{array}$ & $\begin{array}{l}1.292 \\
1.003 \\
6.853-02 \\
3.545\end{array}$ \\
\hline \multirow[t]{2}{*}{1.40} & $\begin{array}{r}0 \\
15 \\
30 \\
40 \\
50\end{array}$ & $\begin{array}{l}9.381-02 \\
8.858 \\
7.314 \\
5.777 \\
3.956\end{array}$ & $\begin{array}{l}9.381-02 \\
8.885 \\
7.411 \\
5.928 \\
4.149\end{array}$ & $\begin{array}{l}1.792-01 \\
1.731 \\
1.543 \\
1.341 \\
1.072\end{array}$ & $\begin{array}{l}1.792-01 \\
1.734 \\
1.556 \\
1.363 \\
1.105\end{array}$ & $\begin{array}{l}2.361-01 \\
2.303 \\
2.122 \\
1.919 \\
1.637\end{array}$ & $\begin{array}{l}2.361-01 \\
2.306 \\
2.134 \\
1.941 \\
1.672\end{array}$ & $\begin{array}{l}2.787-01 \\
2.735 \\
2.568 \\
2.379 \\
2.109\end{array}$ & $\begin{array}{l}2.787-01 \\
2.737 \\
2.579 \\
2.399 \\
2.141\end{array}$ \\
\hline & $\begin{array}{l}60 \\
70 \\
75 \\
80 \\
85\end{array}$ & $\begin{array}{l}2.104 \\
6.673-03 \\
2.637 \\
8.810-04 \\
5.183\end{array}$ & $\begin{array}{l}2.301 \\
8.117-03 \\
3.665 \\
1.575 \\
1.094\end{array}$ & $\begin{array}{l}7.408-02 \\
3.800 \\
2.167 \\
9.249-03 \\
3.270\end{array}$ & $\begin{array}{l}7 \cdot 828-02 \\
4 \cdot 245 \\
2.566 \\
1 \cdot 230 \\
5.333-03\end{array}$ & $\begin{array}{l}1.262 \\
7.942-02 \\
5.400 \\
2.965 \\
1.109\end{array}$ & $\begin{array}{l}1.311 \\
8.572-02 \\
6.054 \\
3.562 \\
1.539\end{array}$ & $\begin{array}{l}1.734 \\
1.230 \\
9.302-02 \\
6.064 \\
2.827\end{array}$ & $\begin{array}{l}1.782 \\
1.300 \\
1.011 \\
6.914-02 \\
3.552\end{array}$ \\
\hline \multirow[t]{3}{*}{6.40} & $\begin{array}{l}0 \\
15 \\
30 \\
40 \\
50\end{array}$ & $\begin{array}{l}1.044-01 \\
9.898-02 \\
8.278 \\
6.642 \\
4.670\end{array}$ & $\begin{array}{l}1.044-01 \\
9.927-02 \\
8.385 \\
6.814 \\
4.892\end{array}$ & $\begin{array}{l}1.891-01 \\
1.830 \\
1.642 \\
1.438 \\
1.165\end{array}$ & $\begin{array}{l}1.891-01 \\
1.834 \\
1.656 \\
1.462 \\
1.200\end{array}$ & $\begin{array}{l}2.439-01 \\
2.382 \\
2.202 \\
2.002 \\
1.72 .1\end{array}$ & $\begin{array}{l}2.439-01 \\
2.385 \\
2.215 \\
2.024 \\
1.756\end{array}$ & $\begin{array}{l}2.844-01 \\
2.792 \\
2.629 \\
2.442 \\
2.175\end{array}$ & $\begin{array}{l}2.844-01 \\
2.795 \\
2.639 \\
2.462 \\
2.207\end{array}$ \\
\hline & 60 & 2.598 & 2.833 & $8.238-02$ & $8.690-02$ & 1.345 & 1.396 & 1.804 & 1.853 \\
\hline & $\begin{array}{l}70 \\
75 \\
80 \\
85\end{array}$ & $\begin{array}{l}8.956-03 \\
3.734 \\
1.203 \\
5.417-04\end{array}$ & $\begin{array}{l}1.075 \\
5.022-03 \\
2.017 \\
1.095\end{array}$ & $\begin{array}{l}4.417 \\
2.617 \\
1.172 \\
3.811-03\end{array}$ & $\begin{array}{l}4.910 \\
3.069 \\
1.520 \\
5.970-03\end{array}$ & $\begin{array}{l}8.694-02 \\
6.058 \\
3.458 \\
1.317\end{array}$ & $\begin{array}{l}9.356-02 \\
6.756 \\
4.107 \\
1.778\end{array}$ & $\begin{array}{l}1.301 \\
9.989-02 \\
6.684 \\
3.237\end{array}$ & $\begin{array}{l}1.373 \\
1.082 \\
7.573-02 \\
4.006\end{array}$ \\
\hline
\end{tabular}




\begin{tabular}{|c|c|c|c|c|c|c|c|c|c|}
\hline \multirow{4}{*}{$\frac{(m b)}{18.4}$} & \multirow{2}{*}{$\begin{array}{r}\theta \\
0 \\
15 \\
30 \\
40 \\
50\end{array}$} & \multicolumn{2}{|c|}{$\lambda=3075.0 \AA$} & \multicolumn{2}{|c|}{$\lambda=3125.0 \AA$} & \multicolumn{2}{|c|}{$\lambda=3175.0 \AA$} & \multicolumn{2}{|c|}{$\lambda=3225.0 \AA$} \\
\hline & & $\begin{array}{l}1.384-01 \\
1.325 \\
1.146 \\
9.592-02 \\
7.227\end{array}$ & $\begin{array}{l}1.384-01 \\
1.329 \\
1.160 \\
9.828-02 \\
7.553\end{array}$ & $\begin{array}{l}2.178-01 \\
2.119 \\
1.933 \\
1.729 \\
1.450\end{array}$ & $\begin{array}{l}2.178-01 \\
2.122 \\
1.949 \\
1.756 \\
1.491\end{array}$ & $\begin{array}{l}2.654-01 \\
2.600 \\
2.428 \\
2.235 \\
1.961\end{array}$ & $\begin{array}{l}2.654-01 \\
2.603 \\
2.441 \\
2.258 \\
1.999\end{array}$ & $\begin{array}{l}2.996-01 \\
2.948 \\
2.792 \\
2.614 \\
2.358\end{array}$ & $\begin{array}{l}2.996-01 \\
2.950 \\
2.802 \\
2.634 \\
2.390\end{array}$ \\
\hline & 60 & 4.535 & 4.916 & 1.089 & 1.145 & 1.589 & 1.645 & 1.999 & 2.049 \\
\hline & $\begin{array}{l}70 \\
75 \\
80 \\
85\end{array}$ & $\begin{array}{l}1.974 \\
9.983-03 \\
3.811 \\
1.460\end{array}$ & $\begin{array}{l}2.317 \\
1.273 \\
5.670-03 \\
2.645\end{array}$ & $\begin{array}{l}6.582-02 \\
4.357 \\
2.326 \\
8.669-03\end{array}$ & $\begin{array}{l}7.246-02 \\
5.015 \\
2.894 \\
1.248\end{array}$ & $\begin{array}{l}1.103 \\
8.227-02 \\
5.284 \\
2.447\end{array}$ & $\begin{array}{l}1.180 \\
9.079-02 \\
6.148 \\
3.143\end{array}$ & $\begin{array}{l}1.507 \\
1.206 \\
8.695-02 \\
4.927\end{array}$ & $\begin{array}{l}1.582 \\
1.297 \\
9.731-02 \\
5.926\end{array}$ \\
\hline 32.8 & $\begin{array}{r}0 \\
15 \\
30 \\
40 \\
50\end{array}$ & $\begin{array}{l}1.735-01 \\
1.674 \\
1.487 \\
1.287 \\
1.024\end{array}$ & $\begin{array}{l}1.735-01 \\
1.679 \\
1.505 \\
1.317 \\
1.068\end{array}$ & $\begin{array}{l}2.442-01 \\
2.385 \\
2.206 \\
2.007 \\
1.731\end{array}$ & $\begin{array}{l}2 \cdot 442-01 \\
2 \cdot 389 \\
2 \cdot 223 \\
2 \cdot 036 \\
1 \cdot 776\end{array}$ & $\begin{array}{l}2.845-01 \\
2.793 \\
2.631 \\
2.446 \\
2.184\end{array}$ & $\begin{array}{l}2.845-01 \\
2.797 \\
2.644 \\
2.470 \\
2.223\end{array}$ & $\begin{array}{l}3.130-01 \\
3.084 \\
2.937 \\
2.768 \\
2.523\end{array}$ & $\begin{array}{l}3.130-01 \\
3.087 \\
2.947 \\
2.786 \\
2.554\end{array}$ \\
\hline & 60 & $7.077-02$ & $7.626-02$ & 1.364 & 1.429 & 1.822 & 1.881 & 2.178 & 2.228 \\
\hline & $\begin{array}{l}70 \\
75 \\
80 \\
85\end{array}$ & $\begin{array}{l}3.713 \\
2.213 \\
1.046 \\
3.909-03\end{array}$ & $\begin{array}{l}4.284 \\
2.723 \\
1.434 \\
6.321-03\end{array}$ & $\begin{array}{l}9.074-02 \\
6.564 \\
4.069 \\
1.853\end{array}$ & $\begin{array}{l}9.902-02 \\
7.442 \\
4.907 \\
2.488\end{array}$ & $\begin{array}{l}1.340 \\
1.054 \\
7.453-02 \\
4.149\end{array}$ & $\begin{array}{l}1.425 \\
1.153 \\
8.521-02 \\
5.124\end{array}$ & $\begin{array}{l}1.701 \\
1.407 \\
1.078 \\
6.971-02\end{array}$ & $\begin{array}{l}1.779 \\
1.503 \\
1.193 \\
8.188-02\end{array}$ \\
\hline 56.0 & $\begin{array}{l}0 \\
15 \\
30 \\
40 \\
50\end{array}$ & $\begin{array}{l}2.154-01 \\
2.094 \\
1.909 \\
1.706 \\
1.431\end{array}$ & $\begin{array}{l}2.154-01 \\
2.100 \\
1.930 \\
1.743 \\
1.487\end{array}$ & $\begin{array}{l}2.733-01 \\
2.680 \\
2.512 \\
2.324 \\
2.059\end{array}$ & $\begin{array}{l}2.733-01 \\
2.684 \\
2.529 \\
2.354 \\
2.107\end{array}$ & $\begin{array}{l}3.051-01 \\
3.004 \\
2.853 \\
2.680 \\
2.433\end{array}$ & $\begin{array}{l}3.051-01 \\
3.007 \\
2.865 \\
2.703 \\
2.471\end{array}$ & $\begin{array}{l}3.277-01 \\
3.234 \\
3.096 \\
2.938 \\
2.707\end{array}$ & $\begin{array}{l}3.277-01 \\
3.236 \\
3.105 \\
2.955 \\
2.736\end{array}$ \\
\hline & 60 & 1.083 & 1.158 & 1.701 & 1.772 & 2.089 & 2.148 & 2.380 & 2.427 \\
\hline & $\begin{array}{l}70 \\
75 \\
80 \\
85\end{array}$ & $\begin{array}{l}6.784-02 \\
4.745 \\
2.882 \\
1.407\end{array}$ & $\begin{array}{l}7.686-02 \\
5.653 \\
3.704 \\
2.026\end{array}$ & $\begin{array}{l}1.239 \\
9.757-02 \\
7.003 \\
4.186\end{array}$ & $\begin{array}{l}1 \cdot 338 \\
1 \cdot 087 \\
8 \cdot 194-02 \\
5 \cdot 289\end{array}$ & $\begin{array}{l}1.624 \\
1.346 \\
1.043 \\
7.065-02\end{array}$ & $\begin{array}{l}1.714 \\
1.454 \\
1.169 \\
8.405-02\end{array}$ & $\begin{array}{l}1.926 \\
1.646 \\
1.335 \\
9.842-02\end{array}$ & $\begin{array}{l}2.002 \\
1.743 \\
1.457 \\
1.127\end{array}$ \\
\hline 132 & $\begin{array}{l}0 \\
15 \\
30 \\
40 \\
50\end{array}$ & $\begin{array}{l}2.761-01 \\
2.708 \\
2.540 \\
2.353 \\
2.093\end{array}$ & $\begin{array}{l}2.761-01 \\
2.713 \\
2.563 \\
2.393 \\
2.155\end{array}$ & $\begin{array}{l}3.151-01 \\
3.105 \\
2.960 \\
2.795 \\
2.558\end{array}$ & $\begin{array}{l}3.151-01 \\
3.109 \\
2.974 \\
2.821 \\
2.601\end{array}$ & $\begin{array}{l}3.366-01 \\
3.325 \\
3.194 \\
3.043 \\
2.824\end{array}$ & $\begin{array}{l}3.366-01 \\
3.327 \\
3.203 \\
3.060 \\
2.853\end{array}$ & $\begin{array}{l}3.521-01 \\
3.484 \\
3.363 \\
3.223 \\
3.018\end{array}$ & $\begin{array}{l}3.521-01 \\
3.485 \\
3.369 \\
3.234 \\
3.037\end{array}$ \\
\hline & $\begin{array}{l}60 \\
70 \\
75 \\
80 \\
85\end{array}$ & $\begin{array}{l}1.745 \\
1.311 \\
1.074 \\
8.391-02 \\
6.066\end{array}$ & $\begin{array}{l}1.836 \\
1.435 \\
1.214 \\
9.907-02 \\
7.577\end{array}$ & $\begin{array}{l}2.231 \\
1.795 \\
1.541 \\
1.276 \\
1.001\end{array}$ & $\begin{array}{l}2.297 \\
1.894 \\
1.661 \\
1.418 \\
1.161\end{array}$ & $\begin{array}{l}2.513 \\
2.086 \\
1.828 \\
1.552 \\
1.266\end{array}$ & $\begin{array}{l}2.560 \\
2.162 \\
1.925 \\
1.675 \\
1.416\end{array}$ & $\begin{array}{l}2.722 \\
2.304 \\
2.044 \\
1.762 \\
1.471\end{array}$ & $\begin{array}{l}2.754 \\
2.361 \\
2.121 \\
1.866 \\
1.608\end{array}$ \\
\hline 370 & $\begin{array}{r}0 \\
15 \\
30 \\
40 \\
50\end{array}$ & $\begin{array}{l}3.511-01 \\
3.471 \\
3.344 \\
3.197 \\
2.982\end{array}$ & $\begin{array}{l}3.511-01 \\
3.474 \\
3.356 \\
3.219 \\
3.019\end{array}$ & $\begin{array}{l}3.757-01 \\
3.725 \\
3.618 \\
3.494 \\
3.309\end{array}$ & $\begin{array}{l}3.757-01 \\
3.726 \\
3.622 \\
3.502 \\
3.323\end{array}$ & $\begin{array}{l}3.892-01 \\
3.863 \\
3.768 \\
3.657 \\
3.489\end{array}$ & $\begin{array}{l}3.892-01 \\
3.863 \\
3.768 \\
3.657 \\
3.491\end{array}$ & $\begin{array}{l}3.990-01 \\
3.963 \\
3.877 \\
3.774 \\
3.618\end{array}$ & $\begin{array}{l}3.990-01 \\
3.963 \\
3.874 \\
3.770 \\
3.612\end{array}$ \\
\hline & 60 & 2.676 & 2.734 & 3.038 & 3.062 & 3.238 & $3 \cdot 244$ & 3.382 & 3.376 \\
\hline & $\begin{array}{l}70 \\
75 \\
80 \\
85\end{array}$ & $\begin{array}{l}2.251 \\
1.995 \\
1.732 \\
1.503\end{array}$ & $\begin{array}{l}2.337 \\
2.101 \\
1.862 \\
1.656\end{array}$ & $\begin{array}{l}2.644 \\
2 \cdot 393 \\
2.121 \\
1.869\end{array}$ & $\begin{array}{l}2.686 \\
2.450 \\
2.199 \\
1.974\end{array}$ & $\begin{array}{l}2.862 \\
2.614 \\
2.336 \\
2.069\end{array}$ & $\begin{array}{l}2.878 \\
2.642 \\
2.382 \\
2.143\end{array}$ & $\begin{array}{l}3.019 \\
2.774 \\
2.490 \\
2.208\end{array}$ & $\begin{array}{l}3.018 \\
2.781 \\
2.512 \\
2.259\end{array}$ \\
\hline
\end{tabular}


Parameters for computing the reflected component of the Upward Radiation

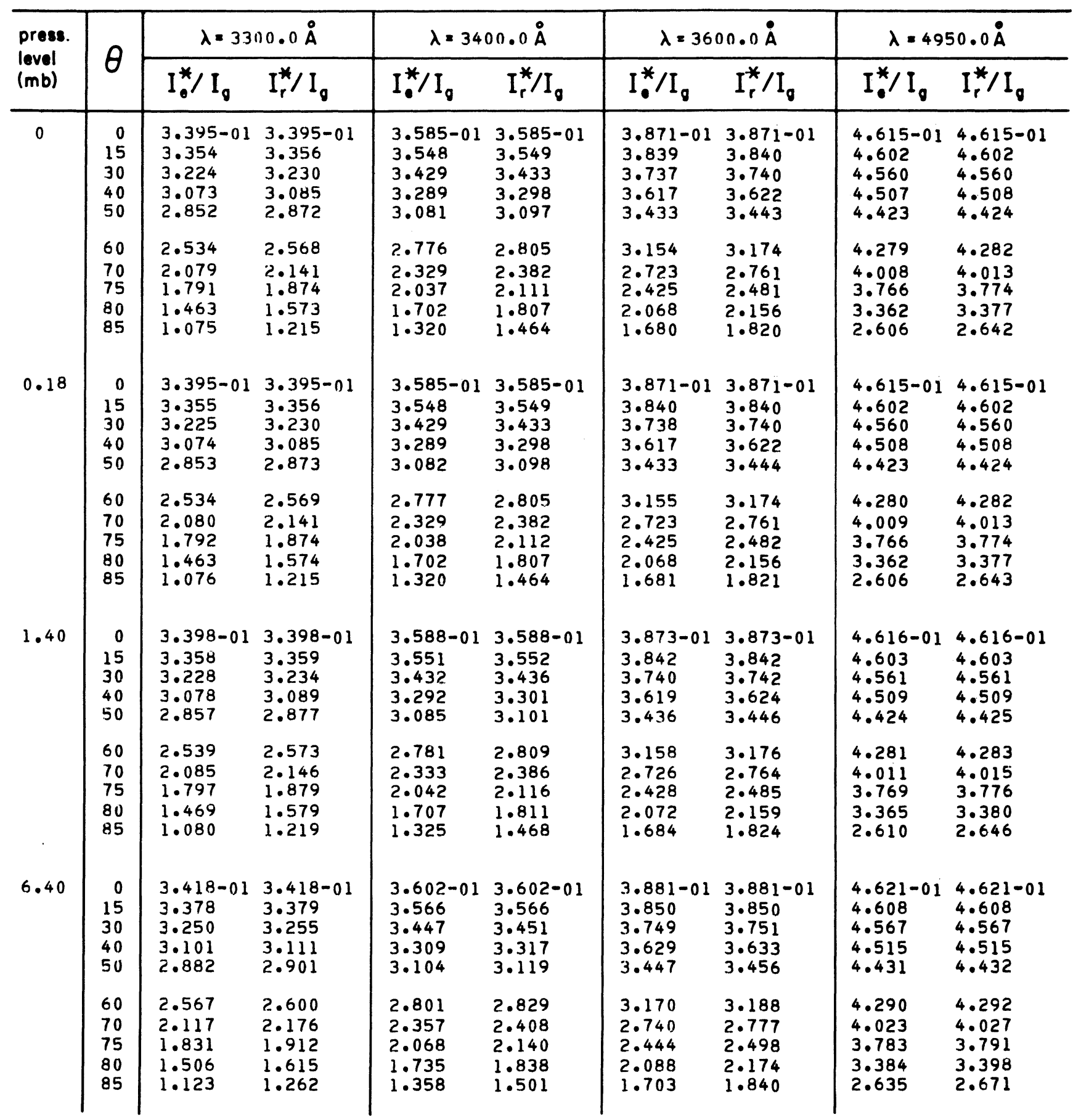




\begin{tabular}{|c|c|c|c|c|c|c|c|c|c|}
\hline$(m b)$ & $\theta$ & $\lambda=330$ & $00.0 \AA$ & $\lambda=340$ & $00.0 \AA$ & $\lambda=360$ & $00.0 \AA$ & $\lambda=495$ & $50.0 \AA$ \\
\hline \multirow[t]{3}{*}{18.4} & $\begin{array}{r}0 \\
15 \\
30 \\
40 \\
50\end{array}$ & $\begin{array}{l}3.467-01 \\
3.429 \\
3.304 \\
3.159 \\
2.947\end{array}$ & $\begin{array}{l}3.467-01 \\
3.430 \\
3.309 \\
3.169 \\
2.964\end{array}$ & $\begin{array}{l}3.637-01 \\
3.602 \\
3.486 \\
3.351 \\
3.150\end{array}$ & $\begin{array}{l}3.637-01 \\
3.602 \\
3.490 \\
3.358 \\
3.164\end{array}$ & $\begin{array}{l}3.900-01 \\
3.870 \\
3.770 \\
3.652 \\
3.473\end{array}$ & $\begin{array}{l}3.900-01 \\
3.870 \\
3.772 \\
3.656 \\
3.481\end{array}$ & $\begin{array}{l}4.633-01 \\
4.621 \\
4.581 \\
4.531 \\
4.450\end{array}$ & $\begin{array}{l}4.633-01 \\
4.621 \\
4.581 \\
4.531 \\
4.451\end{array}$ \\
\hline & 60 & 2.639 & 2.670 & 2.854 & 2.879 & 3.200 & 3.216 & 4.313 & 4.315 \\
\hline & $\begin{array}{l}70 \\
75 \\
80 \\
85\end{array}$ & $\begin{array}{l}2.200 \\
1.922 \\
1.608 \\
1.249\end{array}$ & $\begin{array}{l}2.256 \\
1.999 \\
1.715 \\
1.390\end{array}$ & $\begin{array}{l}2.418 \\
2.134 \\
1.809 \\
1.452\end{array}$ & $\begin{array}{l}2.466 \\
2.202 \\
1.909 \\
1.592\end{array}$ & $\begin{array}{l}2.775 \\
2.481 \\
2.128 \\
1.749\end{array}$ & $\begin{array}{l}2.808 \\
2.532 \\
2.2 .09 \\
1.881\end{array}$ & $\begin{array}{l}4.054 \\
3.821 \\
3.433 \\
2.703\end{array}$ & $\begin{array}{l}4.057 \\
3.827 \\
3.445 \\
2.736\end{array}$ \\
\hline \multirow[t]{3}{*}{32.8} & $\begin{array}{l}0 \\
15 \\
30 \\
40 \\
50\end{array}$ & $\begin{array}{l}3.515-01 \\
3.478 \\
3.357 \\
3.216 \\
3.009\end{array}$ & $\begin{array}{l}3.515-01 \\
3.479 \\
3.361 \\
3.224 \\
3.024\end{array}$ & $\begin{array}{l}3.673-01 \\
3.638 \\
3.526 \\
3.394 \\
3.197\end{array}$ & $\begin{array}{l}3.673-01 \\
3.639 \\
3.528 \\
3.400 \\
3.209\end{array}$ & $\begin{array}{l}3.921-01 \\
3.892 \\
3.794 \\
3.678 \\
3.502\end{array}$ & $\begin{array}{l}3.921-01 \\
3.892 \\
3.796 \\
3.681 \\
3.509\end{array}$ & $\begin{array}{l}4.645-01 \\
4.633 \\
4.594 \\
4.546 \\
4.467\end{array}$ & $\begin{array}{l}4.645-01 \\
4.633 \\
4.594 \\
4.546 \\
4.468\end{array}$ \\
\hline & 60 & 2.709 & 2.737 & 2.907 & 2.929 & 3.233 & 3.247 & 4.335 & 4.336 \\
\hline & $\begin{array}{l}70 \\
75 \\
80 \\
85\end{array}$ & $\begin{array}{l}2.279 \\
2.008 \\
1.704 \\
1.368\end{array}$ & $\begin{array}{l}2.332 \\
2.081 \\
1.807 \\
1.508\end{array}$ & $\begin{array}{l}2.479 \\
2.199 \\
1.882 \\
1.540\end{array}$ & $\begin{array}{l}2.522 \\
2.263 \\
1.976 \\
1.677\end{array}$ & $\begin{array}{l}2.813 \\
2.522 \\
2.171 \\
1.797\end{array}$ & $\begin{array}{l}2.843 \\
2.568 \\
2.247 \\
1.922\end{array}$ & $\begin{array}{l}4.083 \\
3.857 \\
3.479 \\
2.765\end{array}$ & $\begin{array}{l}4.086 \\
3.862 \\
3.489 \\
2.795\end{array}$ \\
\hline \multirow[t]{3}{*}{56.0} & $\begin{array}{r}0 \\
15 \\
30 \\
40 \\
50\end{array}$ & $\begin{array}{l}3.576-01 \\
3.540 \\
3.424 \\
3.288 \\
3.088\end{array}$ & $\begin{array}{l}3.576-01 \\
3.541 \\
3.427 \\
3.295 \\
3.101\end{array}$ & $\begin{array}{l}3.721-01 \\
3.687 \\
3.579 \\
3.451 \\
3.261\end{array}$ & $\begin{array}{l}3.721-01 \\
3.688 \\
3.581 \\
3.455 \\
3.269\end{array}$ & $\begin{array}{l}3.954-01 \\
3.925 \\
3.830 \\
3.717 \\
3.546\end{array}$ & $\begin{array}{l}3.954-01 \\
3.925 \\
3.831 \\
3.719 \\
3.550\end{array}$ & $\begin{array}{l}4.659-01 \\
4.648 \\
4.611 \\
4.564 \\
4.489\end{array}$ & $\begin{array}{l}4.659-01 \\
4.648 \\
4.611 \\
4.564 \\
4.489\end{array}$ \\
\hline & 60 & 2.797 & 2.821 & 2.978 & 2.996 & 3.283 & 3.293 & 4.362 & 4.362 \\
\hline & $\begin{array}{l}70 \\
75 \\
80 \\
85\end{array}$ & $\begin{array}{l}2.379 \\
2.115 \\
1.822 \\
1.511\end{array}$ & $\begin{array}{l}2.425 \\
2.181 \\
1.917 \\
1.646\end{array}$ & $\begin{array}{l}2.559 \\
2.285 \\
1.974 \\
1.650\end{array}$ & $\begin{array}{l}2.597 \\
2.341 \\
2.060 \\
1.779\end{array}$ & $\begin{array}{l}2.870 \\
2.582 \\
2.234 \\
1.864\end{array}$ & $\begin{array}{l}2.895 \\
2.621 \\
2.301 \\
1.979\end{array}$ & $\begin{array}{l}4.120 \\
3.901 \\
3.535 \\
2.838\end{array}$ & $\begin{array}{l}4.121 \\
3.904 \\
3.542 \\
2.864\end{array}$ \\
\hline \multirow[t]{3}{*}{132} & $\begin{array}{l}0 \\
15 \\
30 \\
40 \\
50\end{array}$ & $\begin{array}{l}3.725-01 \\
3.692 \\
3.587 \\
3.464 \\
3.280\end{array}$ & $\begin{array}{l}3.725-01 \\
3.692 \\
3.587 \\
3.465 \\
3.284\end{array}$ & $\begin{array}{l}3.848-01 \\
3.818 \\
3.719 \\
3.603 \\
3.428\end{array}$ & $\begin{array}{l}3.848-01 \\
3.818 \\
3.719 \\
3.603 \\
3.429\end{array}$ & $\begin{array}{l}4.052-01 \\
4.025 \\
3.939 \\
3.836 \\
3.678\end{array}$ & $\begin{array}{l}4.052-01 \\
4.025 \\
3.938 \\
3.834 \\
3.676\end{array}$ & $\begin{array}{l}4.694-01 \\
4.684 \\
4.650 \\
4.609 \\
4.541\end{array}$ & $\begin{array}{l}4.694-01 \\
4.684 \\
4.650 \\
4.608 \\
4.539\end{array}$ \\
\hline & 60 & 3.010 & 3.019 & 3.165 & 3.170 & 3.433 & 3.433 & 4.426 & 4.424 \\
\hline & $\begin{array}{l}70 \\
75 \\
80 \\
85\end{array}$ & $\begin{array}{l}2.613 \\
2.359 \\
2.075 \\
1.790\end{array}$ & $\begin{array}{l}2.638 \\
2.399 \\
2.141 \\
1.893\end{array}$ & $\begin{array}{l}2.767 \\
2.502 \\
2.197 \\
1.887\end{array}$ & $\begin{array}{l}2.785 \\
2.533 \\
2.253 \\
1.983\end{array}$ & $\begin{array}{l}3.042 \\
2.762 \\
2.418 \\
2.048\end{array}$ & $\begin{array}{l}3.050 \\
2.781 \\
2.457 \\
2.130\end{array}$ & $\begin{array}{l}4.205 \\
4.004 \\
3.662 \\
2.994\end{array}$ & $\begin{array}{l}4.202 \\
4.001 \\
3.661 \\
3.005\end{array}$ \\
\hline \multirow[t]{3}{*}{370} & $\begin{array}{r}0 \\
15 \\
30 \\
40 \\
50\end{array}$ & $\begin{array}{l}4.116-01 \\
4.093 \\
4.018 \\
3.927 \\
3.788\end{array}$ & $\begin{array}{l}4.116-01 \\
4.092 \\
4.011 \\
3.916 \\
3.772\end{array}$ & $\begin{array}{l}4.199-01 \\
4.178 \\
4.108 \\
4.023 \\
3.892\end{array}$ & $\begin{array}{l}4.199-01 \\
4.177 \\
4.102 \\
4.012 \\
3.875\end{array}$ & $\begin{array}{l}4.338-01 \\
4.320 \\
4.259 \\
4.185 \\
4.068\end{array}$ & $\begin{array}{l}4.338-01 \\
4.318 \\
4.253 \\
4.175 \\
4.052\end{array}$ & $\begin{array}{l}4.784-01 \\
4.777 \\
4.754 \\
4.724 \\
4.676\end{array}$ & $\begin{array}{l}4.784-01 \\
4.777 \\
4.752 \\
4.722 \\
4.672\end{array}$ \\
\hline & 60 & 3.574 & 3.551 & 3.686 & 3.663 & 3.880 & 3.857 & 4.593 & 4.587 \\
\hline & $\begin{array}{l}70 \\
75 \\
80 \\
85\end{array}$ & $\begin{array}{l}3.234 \\
2.995 \\
2.707 \\
2.408\end{array}$ & $\begin{array}{l}3.208 \\
2.972 \\
2.693 \\
2.421\end{array}$ & $\begin{array}{l}3.351 \\
3.107 \\
2.799 \\
2.466\end{array}$ & $\begin{array}{l}3.322 \\
3.078 \\
2.779 \\
2.472\end{array}$ & $\begin{array}{l}3.560 \\
3.312 \\
2.976 \\
2.568\end{array}$ & $\begin{array}{l}3.530 \\
3.280 \\
2.947 \\
2.562\end{array}$ & $\begin{array}{l}4.431 \\
4.278 \\
4.007 \\
3.417\end{array}$ & $\begin{array}{l}4.420 \\
4.264 \\
3.988 \\
3.393\end{array}$ \\
\hline
\end{tabular}


Parameters for computing the reflected component of the Upward Radiation

\begin{tabular}{|c|c|c|c|c|c|c|c|c|c|}
\hline \multirow{2}{*}{$\begin{array}{l}\text { press. } \\
\text { level } \\
\text { (mb) }\end{array}$} & \multirow{2}{*}{$\theta$} & \multicolumn{2}{|c|}{$\lambda=5350.0 \AA$} & \multicolumn{2}{|c|}{$\lambda=5750.0 \AA$} & \multicolumn{2}{|c|}{$\lambda=6150.0 \AA$} & \multicolumn{2}{|c|}{$\lambda=6550.0 \AA$} \\
\hline & & $I_{0}^{*} / I_{g}$ & $I_{r}^{*} / I_{g}$ & $I_{0}^{*} / I_{0}$ & $I_{p}^{*} / I_{q}$ & $I_{0}^{*} / I_{g}$ & $I_{p}^{*} / I_{g}$ & $I_{*}^{*} / I_{0}$ & \\
\hline 0 & $\begin{array}{r}0 \\
15 \\
30 \\
40 \\
50\end{array}$ & $\begin{array}{l}4.625-01 \\
4.612 \\
4.570 \\
4.518 \\
4.433\end{array}$ & $\begin{array}{l}4.625-01 \\
4.612 \\
4.570 \\
4.518 \\
4.434\end{array}$ & $\begin{array}{l}4.611-01 \\
4.598 \\
4.554 \\
4.499 \\
4.410\end{array}$ & $\begin{array}{l}4.611-01 \\
4.598 \\
4.554 \\
4.500 \\
4.412\end{array}$ & $\begin{array}{l}4.665-01 \\
4.654 \\
4.616 \\
4.568 \\
4.490\end{array}$ & $\begin{array}{l}4.665-01 \\
4.654 \\
4.616 \\
4.569 \\
4.491\end{array}$ & $\begin{array}{l}4.784-01 \\
4.777 \\
4.752 \\
4.720 \\
4.669\end{array}$ & $\begin{array}{l}4.784-01 \\
4.777 \\
4.752 \\
4.721 \\
4.670\end{array}$ \\
\hline & 60 & $4 \cdot 288$ & 4.290 & $4 \cdot 258$ & $4 \cdot 260$ & $4 \cdot 356$ & 4.358 & 4.580 & 4.581 \\
\hline & $\begin{array}{l}70 \\
75 \\
80 \\
85\end{array}$ & $\begin{array}{l}4.009 \\
3.753 \\
3.310 \\
2.400\end{array}$ & $\begin{array}{l}4.013 \\
3.760 \\
3.322 \\
2.426\end{array}$ & $\begin{array}{l}3.962 \\
3.688 \\
3.207 \\
2.186\end{array}$ & $\begin{array}{l}3.966 \\
3.694 \\
3.216 \\
2.204\end{array}$ & $\begin{array}{l}4.093 \\
3.845 \\
3.400 \\
2.403\end{array}$ & $\begin{array}{l}4.096 \\
3.849 \\
3.407 \\
2.416\end{array}$ & $\begin{array}{l}4.401 \\
4.229 \\
3.909 \\
3.124\end{array}$ & $\begin{array}{l}4.403 \\
4.231 \\
3.912 \\
3.131\end{array}$ \\
\hline 0.18 & $\begin{array}{l}0 \\
15 \\
30 \\
40 \\
50\end{array}$ & $\begin{array}{l}4.625-01 \\
4.612 \\
4.570 \\
4.518 \\
4.433\end{array}$ & $\begin{array}{l}4.625-01 \\
4.612 \\
4.571 \\
4.519 \\
4.434\end{array}$ & $\begin{array}{l}4.611-01 \\
4.598 \\
4.554 \\
4.499 \\
4.410\end{array}$ & $\begin{array}{l}4.611-01 \\
4.598 \\
4.555 \\
4.500 \\
4.412\end{array}$ & $\begin{array}{l}4.665-01 \\
4.654 \\
4.616 \\
4.568 \\
4.490\end{array}$ & $\begin{array}{l}4.665-01 \\
4.654 \\
4.616 \\
4.569 \\
4.491\end{array}$ & $\begin{array}{l}4.784-01 \\
4.777 \\
4.752 \\
4.721 \\
4.669\end{array}$ & $\begin{array}{l}4.784-01 \\
4.777 \\
4.752 \\
4.721 \\
4.670\end{array}$ \\
\hline & $\begin{array}{l}60 \\
70 \\
75 \\
80 \\
85\end{array}$ & $\begin{array}{l}4.288 \\
4.009 \\
3.753 \\
3.311 \\
2.401\end{array}$ & $\begin{array}{l}4.290 \\
4.014 \\
3.760 \\
3.322 \\
2.426\end{array}$ & $\begin{array}{l}4.258 \\
3.962 \\
3.688 \\
3.207 \\
2.187\end{array}$ & $\begin{array}{l}4.260 \\
3.966 \\
3.694 \\
3.217 \\
2.205\end{array}$ & $\begin{array}{l}4.356 \\
4.093 \\
3.845 \\
3.401 \\
2.404\end{array}$ & $\begin{array}{l}4.358 \\
4.096 \\
3.850 \\
3.407 \\
2.417\end{array}$ & $\begin{array}{l}4.580 \\
4.401 \\
4.229 \\
3.909 \\
3.124\end{array}$ & $\begin{array}{l}4.581 \\
4.403 \\
4.231 \\
3.912 \\
3.132\end{array}$ \\
\hline 1.40 & $\begin{array}{r}0 \\
15 \\
30 \\
40 \\
50\end{array}$ & $\begin{array}{l}4.626-01 \\
4.614 \\
4.572 \\
4.520 \\
4.435\end{array}$ & $\begin{array}{l}4.626-01 \\
4.614 \\
4.572 \\
4.520 \\
4.436\end{array}$ & $\begin{array}{l}4.613-01 \\
4.600 \\
4.557 \\
4.502 \\
4.414\end{array}$ & $\begin{array}{l}4.613-01 \\
4.600 \\
4.557 \\
4.503 \\
4.415\end{array}$ & $\begin{array}{l}4.667-01 \\
4.656 \\
4.618 \\
4.571 \\
4.493\end{array}$ & $\begin{array}{l}4.667-01 \\
4.656 \\
4.618 \\
4.571 \\
4.494\end{array}$ & $\begin{array}{l}4.785-01 \\
4.778 \\
4.753 \\
4.722 \\
4.671\end{array}$ & $\begin{array}{l}4.785-01 \\
4.778 \\
4.753 \\
4.722 \\
4.671\end{array}$ \\
\hline & $\begin{array}{l}60 \\
70 \\
75 \\
80 \\
85\end{array}$ & $\begin{array}{l}4.291 \\
4.013 \\
3.758 \\
3.316 \\
2.408\end{array}$ & $\begin{array}{l}4.293 \\
4.017 \\
3.764 \\
3.328 \\
2.433\end{array}$ & $\begin{array}{l}4.262 \\
3.968 \\
3.695 \\
3.216 \\
2.197\end{array}$ & $\begin{array}{l}4.264 \\
3.972 \\
3.701 \\
3.225 \\
2.215\end{array}$ & $\begin{array}{l}4.360 \\
4.098 \\
3.852 \\
3.409 \\
2.415\end{array}$ & $\begin{array}{l}4.362 \\
4.101 \\
3.856 \\
3.415 \\
2.428\end{array}$ & $\begin{array}{l}4.582 \\
4.404 \\
4.233 \\
3.914 \\
3.132\end{array}$ & $\begin{array}{l}4.583 \\
4.406 \\
4.235 \\
3.917 \\
3.140\end{array}$ \\
\hline 6.40 & $\begin{array}{l}0 \\
15 \\
30 \\
40 \\
50\end{array}$ & $\begin{array}{l}4.638-01 \\
4.626 \\
4.585 \\
4.534 \\
4.452\end{array}$ & $\begin{array}{l}4.638-01 \\
4.626 \\
4.585 \\
4.535 \\
4.453\end{array}$ & $\begin{array}{l}4.631-01 \\
4.619 \\
4.577 \\
4.525 \\
4.440\end{array}$ & $\begin{array}{l}4.631-01 \\
4.619 \\
4.577 \\
4.526 \\
4.441\end{array}$ & $\begin{array}{l}4.684-01 \\
4.673 \\
4.637 \\
4.592 \\
4.519\end{array}$ & $\begin{array}{l}4.684-01 \\
4.674 \\
4.638 \\
4.593 \\
4.520\end{array}$ & $\begin{array}{l}4.795-01 \\
4.788 \\
4.764 \\
4.734 \\
4.685\end{array}$ & $\begin{array}{l}4 \cdot 795-01 \\
4.788 \\
4.764 \\
4.734 \\
4.685\end{array}$ \\
\hline & 60 & 4.312 & 4.314 & 4.295 & 4.297 & 4.392 & 4.393 & 4.600 & 4.600 \\
\hline & $\begin{array}{l}70 \\
75 \\
80 \\
85\end{array}$ & $\begin{array}{l}4.042 \\
3.793 \\
3.363 \\
2.473\end{array}$ & $\begin{array}{l}4.046 \\
3.800 \\
3.373 \\
2.497\end{array}$ & $\begin{array}{l}4.012 \\
3.750 \\
3.287 \\
2.292\end{array}$ & $\begin{array}{l}4.016 \\
3.755 \\
3.296 \\
2.310\end{array}$ & $\begin{array}{l}4.141 \\
3.905 \\
3.479 \\
2.514\end{array}$ & $\begin{array}{l}4.144 \\
3.909 \\
3.486 \\
2.526\end{array}$ & $\begin{array}{l}4.429 \\
4.265 \\
3.957 \\
3.201\end{array}$ & $\begin{array}{l}4.431 \\
4.267 \\
3.961 \\
3.208\end{array}$ \\
\hline
\end{tabular}




\begin{tabular}{|c|c|c|c|c|c|c|c|c|c|}
\hline \multirow[b]{2}{*}{18.4} & $\theta$ & \multicolumn{2}{|c|}{$\lambda=5350.0 \AA$} & \multicolumn{2}{|c|}{$\lambda=5750.0 \AA$} & \multicolumn{2}{|c|}{$\lambda=6150.0 \AA$} & \multicolumn{2}{|c|}{$\lambda=6550.0 \AA$} \\
\hline & $\begin{array}{l}0 \\
15 \\
30 \\
40 \\
50 \\
\\
60 \\
70 \\
75 \\
80 \\
85\end{array}$ & $\begin{array}{l}4.668-01 \\
4.656 \\
4.619 \\
4.573 \\
4.497 \\
4.368 \\
4.118 \\
3.888 \\
3.487 \\
2.651\end{array}$ & $\begin{array}{l}4.668-01 \\
4.657 \\
4.619 \\
4.573 \\
4.498 \\
4.370 \\
4.122 \\
3.893 \\
3.497 \\
2.674\end{array}$ & $\begin{array}{l}4.678-01 \\
4.667 \\
4.630 \\
4.585 \\
4.510 \\
4.382 \\
4.132 \\
3.898 \\
3.481 \\
2.565\end{array}$ & $\begin{array}{l}4.678-01 \\
4.667 \\
4.631 \\
4.586 \\
4.512 \\
4.384 \\
4.136 \\
3.903 \\
3.490 \\
2.582\end{array}$ & $\begin{array}{l}4.729-01 \\
4.719 \\
4.688 \\
4.649 \\
4.586 \\
4.475 \\
4.257 \\
4.050 \\
3.673 \\
2.796\end{array}$ & $\begin{array}{l}4.729-01 \\
4.720 \\
4.689 \\
4.650 \\
4.586 \\
4.477 \\
4.260 \\
4.054 \\
3.679 \\
2.808\end{array}$ & $\begin{array}{l}4.819-01 \\
4.813 \\
4.792 \\
4.765 \\
4.722 \\
4.647 \\
4.495 \\
4.349 \\
4.074 \\
3.389\end{array}$ & $\begin{array}{l}4.819-01 \\
4.813 \\
4.792 \\
4.766 \\
4.722 \\
4.647 \\
4.496 \\
4.350 \\
4.077 \\
3.395\end{array}$ \\
\hline 32.8 & $\begin{array}{l}0 \\
15 \\
30 \\
40 \\
50 \\
\\
60 \\
70 \\
75 \\
80 \\
85\end{array}$ & $\begin{array}{l}4.693-01 \\
4.682 \\
4.648 \\
4.605 \\
4.535 \\
4.415 \\
4.183 \\
3.968 \\
3.593 \\
2.806\end{array}$ & $\begin{array}{l}4.693-01 \\
4.682 \\
4.648 \\
4.605 \\
4.536 \\
4.416 \\
4.186 \\
3.973 \\
3.602 \\
2.828\end{array}$ & $\begin{array}{l}4.716-01 \\
4.707 \\
4.674 \\
4.634 \\
4.568 \\
4.454 \\
4.232 \\
4.022 \\
3.646 \\
2.806\end{array}$ & $\begin{array}{l}4.716-01 \\
4.707 \\
4.675 \\
4.635 \\
4.569 \\
4.456 \\
4.235 \\
4.027 \\
3.654 \\
2.823\end{array}$ & $\begin{array}{l}4.765-01 \\
4.757 \\
4.730 \\
4.696 \\
4.640 \\
4.544 \\
4.353 \\
4.170 \\
3.836 \\
3.043\end{array}$ & $\begin{array}{l}4.765-01 \\
4.757 \\
4.730 \\
4.696 \\
4.641 \\
4.545 \\
4.355 \\
4.174 \\
3.841 \\
3.055\end{array}$ & $\begin{array}{l}4.839-01 \\
4.833 \\
4.814 \\
4.791 \\
4.752 \\
4.685 \\
4.550 \\
4.418 \\
4.171 \\
3.548\end{array}$ & $\begin{array}{l}4.839-01 \\
4.833 \\
4.815 \\
4.791 \\
4.753 \\
4.686 \\
4.551 \\
4.419 \\
4.173 \\
3.554\end{array}$ \\
\hline 56.0 & $\begin{array}{l}0 \\
15 \\
30 \\
40 \\
50 \\
\\
60 \\
70 \\
75 \\
80 \\
85\end{array}$ & $\begin{array}{l}4.719-01 \\
4.709 \\
4.678 \\
4.638 \\
4.574 \\
4.464 \\
4.251 \\
4.052 \\
3.705 \\
2.970\end{array}$ & $\begin{array}{l}4.719-01 \\
4.709 \\
4.678 \\
4.639 \\
4.575 \\
4.465 \\
4.252 \\
4.056 \\
3.711 \\
2.989\end{array}$ & $\begin{array}{l}4.754-01 \\
4.746 \\
4.718 \\
4.683 \\
4.625 \\
4.526 \\
4.332 \\
4.147 \\
3.815 \\
3.061\end{array}$ & $\begin{array}{l}4.754-01 \\
4.746 \\
4.718 \\
4.683 \\
4.626 \\
4.528 \\
4.334 \\
4.151 \\
3.821 \\
3.076\end{array}$ & $\begin{array}{l}4.800-01 \\
4.793 \\
4.770 \\
4.741 \\
4.694 \\
4.611 \\
4.448 \\
4.290 \\
4.000 \\
3.302\end{array}$ & $\begin{array}{l}4.800-01 \\
4.793 \\
4.770 \\
4.741 \\
4.694 \\
4.612 \\
4.449 \\
4.293 \\
4.004 \\
3.312\end{array}$ & $\begin{array}{l}4.858-01 \\
4.854 \\
4.837 \\
4.816 \\
4.783 \\
4.723 \\
4.604 \\
4.488 \\
4.268 \\
3.712\end{array}$ & $\begin{array}{l}4.858-01 \\
4.854 \\
4.837 \\
4.817 \\
4.783 \\
4.724 \\
4.604 \\
4.489 \\
4.270 \\
3.716\end{array}$ \\
\hline 132 & $\begin{array}{l}0 \\
15 \\
30 \\
40 \\
50 \\
\\
60 \\
70 \\
75 \\
80 \\
85\end{array}$ & $\begin{array}{l}4.759-01 \\
4.751 \\
4.724 \\
4.690 \\
4.635 \\
4.541 \\
4.356 \\
4.183 \\
3.876 \\
3.212\end{array}$ & $\begin{array}{l}4.759-01 \\
4.751 \\
4.724 \\
4.690 \\
4.635 \\
4.540 \\
4.355 \\
4.182 \\
3.876 \\
3.220\end{array}$ & $\begin{array}{l}4.802-01 \\
4.796 \\
4.773 \\
4.745 \\
4.699 \\
4.619 \\
4.460 \\
4.309 \\
4.034 \\
3.394\end{array}$ & $\begin{array}{l}4.802-01 \\
4.796 \\
4.773 \\
4.745 \\
4.699 \\
4.619 \\
4.461 \\
4.310 \\
4.036 \\
3.401\end{array}$ & $\begin{array}{l}4.843-01 \\
4.838 \\
4.820 \\
4.797 \\
4.760 \\
4.695 \\
4.565 \\
4.439 \\
4.205 \\
3.629\end{array}$ & $\begin{array}{l}4.843-01 \\
4 \cdot 838 \\
4.820 \\
4.797 \\
4.760 \\
4.695 \\
4.565 \\
4.440 \\
4.206 \\
3.634\end{array}$ & $\begin{array}{l}4.884-01 \\
4.880 \\
4.867 \\
4.850 \\
4.822 \\
4.774 \\
4.675 \\
4.579 \\
4.397 \\
3.927\end{array}$ & $\begin{array}{l}4.884-01 \\
4.880 \\
4.867 \\
4.850 \\
4.822 \\
4.773 \\
4.675 \\
4.579 \\
4.397 \\
3.928\end{array}$ \\
\hline 370 & $\begin{array}{l}0 \\
15 \\
30 \\
40 \\
50 \\
\\
60 \\
70 \\
75 \\
80 \\
85\end{array}$ & $\begin{array}{l}4.833-01 \\
4.828 \\
4.809 \\
4.786 \\
4.747 \\
4.681 \\
4.549 \\
4.422 \\
4.189 \\
3.642\end{array}$ & $\begin{array}{l}4.833-01 \\
4.827 \\
4.808 \\
4.784 \\
4.745 \\
4.677 \\
4.542 \\
4.413 \\
4.177 \\
3.625\end{array}$ & $\begin{array}{l}4.867-01 \\
4.862 \\
4.847 \\
4.828 \\
4.797 \\
4.742 \\
4.633 \\
4.526 \\
4.327 \\
3.830\end{array}$ & $\begin{array}{l}4.867-01 \\
4.862 \\
4.847 \\
4.827 \\
4.796 \\
4.740 \\
4.629 \\
4.521 \\
4.320 \\
3.820\end{array}$ & $\begin{array}{l}4.895-01 \\
4.892 \\
4.880 \\
4.864 \\
4.840 \\
4.796 \\
4.707 \\
4.621 \\
4.455 \\
4.025\end{array}$ & $\begin{array}{l}4.895-01 \\
4.892 \\
4.879 \\
4.864 \\
4.839 \\
4.794 \\
4.705 \\
4.617 \\
4.450 \\
4.018\end{array}$ & $\begin{array}{l}4.921-01 \\
4.918 \\
4.909 \\
4.898 \\
4.879 \\
4.845 \\
4.778 \\
4.710 \\
4.580 \\
4.231\end{array}$ & $\begin{array}{l}4.921-01 \\
4.918 \\
4.909 \\
4.897 \\
4.878 \\
4.844 \\
4.776 \\
4.708 \\
4.577 \\
4.225\end{array}$ \\
\hline
\end{tabular}

